\title{
REPRESENTACIONES DE ENFERMERAS COMUNITARIAS SOBRE ASPECTOS ÉTICOS Y LEGALES EN EL CUIDADO CON PLANTAS MEDICINALES ${ }^{1}$
}

\author{
REPRESENTATIONS OF COMMUNITY NURSES ABOUT ETHICAL \\ AND LEGAL ASPECTS IN THE CARE WITH MEDICINAL PLANTS
}

\section{REPRESENTAÇÕES DE ENFERMEIROS COMUNITÁRIOS SOBRE ASPECTOS ÉTICOS E LEGAIS NO CUIDADO COM PLANTAS MEDICINAIS}

\author{
LIZET VÉLIZ-RoJAs*
}

\begin{abstract}
RESUMEN
Objetivo: Describir las representaciones sociales de las enfermeras comunitarias sobre los aspectos éticos y legales en el uso terapéutico de plantas medicinales, como parte de los cuidados. Material y Métodos: Estudio cualitativo, descriptivo, hermenéutico, delineado por medio de las Representaciones Sociales en su enfoque procesual. Los métodos de producción de información fueron la entrevista enfocada y dos grupos focales. Se realizó triangulación de fuentes y de datos y se utilizó el análisis de contenido cualitativo inductivo. Resultados: Se presentan seis categorías: Políticas públicas, Prescripción de plantas medicinales, Calidad de los fitofármacos, Cuidado complementario, Cuidado con evidencia y Educación a la comunidad. Conclusión: Emergen dos aspectos centrales: El primero es el desarrollo de políticas públicas que apunten hacia el respaldo del accionar de la profesión y el avance de la prescripción enfermera, que permita la autonomía de la profesión respecto al cuidado con plantas medicinales en la comunidad.
\end{abstract}

Palabras clave: Plantas medicinales; Enfermería en Salud Comunitaria; Ética Profesional; Legislación de Enfermería.

\begin{abstract}
Objective: Describe the social representations of community nurses about ethical and legal aspects of the therapeutic use of medicinal plants. Material and Methods: Qualitative, descriptive and hermeneutical study, guided by the Social Representations in their procedural approach. Methods of information gathering consisted of focused interview and two focus groups. Data and source triangulation were conducted, using inductive qualitative content analysis. Results: Six categories were established: public policies, prescription of medicinal
\end{abstract}

${ }^{1}$ Investigación financiada a través del Proyecto DIDULS Nº 10201020897 de la Dirección de Investigación y Desarrollo Universidad de La Serena - Chile.

*Dra. en Enfermería, Departamento de Enfermería, Universidad de La Serena, Chile. ORCID: https://orcid.org/0000-00028961-1814..Email: lveliz@userena.cl 
plants, quality of phytopharmaceuticals, complementary care, evidence-based health care and community education. Conclusion: Two central aspects emerged: the development of public policies to support the practice of the profession, and the advancement of the nurse prescribing, contributing to autonomy with respect to health care provision with medicinal plants in the community.

Key words: Medicinal plants; Community Health Nursing; Professional Ethics; Nursing Legislation.

\section{RESUMO}

Objetivo: Descrever as representaçóes sociais dos enfermeiros da comunidade sobre aspectos éticos e legais no uso terapêutico de plantas medicinais como parte do cuidado. Material e Métodos: Estudo qualitativo, descritivo, hermenêutico, delineado pelas Representaçôes Sociais em sua abordagem processual. Os métodos de produção da informação foram a entrevista focalizada e dois grupos focais. Foi realizada a triangulação de fontes e dados e a análise qualitativa indutiva de conteúdo. Resultados: Foram apresentadas seis categorias: Políticas públicas, Prescrição de plantas medicinais, Qualidade dos fitofarmacêuticos, Cuidados complementares, Cuidados com evidências e Educação à comunidade. Conclusão: Dois aspectos centrais emergem: $\mathrm{O}$ desenvolvimento de políticas públicas que apontem para o apoio à atividade da profissão e o avanço da prescriçáo do enfermeiro, o que permite a autonomia da profissáo em relaçáo ao cuidado com plantas medicinais na comunidade.

Palavras-chave: Plantas Medicinais; Enfermagem em Saúde Comunitária; Ética Profissional; Legislação de Enfermagem.

Fecha de recepción: 25/10/2019

Fecha de aceptación: 28/07/2020

\section{INTRODUCCIÓN}

El proceso histórico del uso de las plantas medicinales asociadas al cuidado de la salud está orientado a los conocimientos populares traspasados de generación en generación. Se ha reportado que entre el 65 y $80 \%$ de la población utiliza plantas medicinales y en el caso de Chile, el uso de hierbas medicinales ha aumentado durante las últimas tres décadas ${ }^{(1)}$. Autores establecen que con esta tendencia del uso de fitoterapia por parte de la población, los profesionales de la salud no pueden ser ajenos a la utilización de productos naturales, principalmente en las enfermedades crónicas no transmisibles, dada la pandemia presente y la previsión futura ${ }^{(2)}$.

La Organización Mundial de la Salud (OMS) ${ }^{(3)}$ las define como materiales vegetales brutos, tales como hojas, flores, frutos, semillas, tallos, madera, corteza, raíces, rizomas y otras partes de las plantas, enteros, fragmentados o pulverizados, que se utilizan para la prevención, el diagnóstico, la mejora o el tratamiento de enfermedades físicas o mentales. En esta misma línea, la OMS ha promovido y reconocido la importancia de la medicina tradicional y del uso de plantas medicinales para el cuidado de la salud $^{(4)}$.

Las experiencias ancestrales acumuladas en el tiempo, su accesibilidad y su bajo costo, convierten a la medicina herbaria en la alternativa principal para la atención primaria de salud, lo que ha permitido que estas prácticas se mantengan hasta la actualidad, especialmente en sectores rurales ${ }^{(5)}$. Los diversos productos medicinales tradicionales han evolucionado acorde a las características etnológicas, culturales, climáticas, geográficas e incluso filosóficas del contexto donde se encuentran insertas.

En el siglo XX, desde la medicina occidental, se descalificó el conocimiento popular sobre las plantas medicinales, basándose en la inferioridad percibida de la eficacia y seguridad, e imprecisión y limitada objetividad del conocimiento popular de las plantas medicinales, en comparación a los medicamentos sintéticos y el conocimiento científico. Sin embargo, la percepción sobre las limitaciones de la medicina convencional, el aumento de las enfermedades iatrogénicas y crónicas, el debilitamiento de la relación médico-paciente y la 
búsqueda de atención médica integral, además de los problemas de inclusión financiera y social, han llevado a las personas a buscar tratamientos menos agresivos y volver a las terapias naturales ${ }^{(6)}$. En este contexto se han desarrollado diversas políticas para implementar e incorporar el uso de plantas medicinales en los sistemas sanitarios.

En el caso de Chile, en el año 2009, un total de 103 plantas medicinales, denominadas Medicamentos Herbarios Tradicionales (MHT), se incorporaron en el concepto de medicamentos ${ }^{(7,8)}$. La resolución exenta $\mathrm{No}^{\circ} 548^{(9)}$ establece que las plantas medicinales que se encuentran en el listado de MHT están autorizadas para la venta y distribución, una vez que un servicio de salud competente haya autorizado el establecimiento en el que se almacenan, fraccionan, envasan o realizan otras operaciones propias de su procesamiento $\mathrm{y}$, además, deberá estar envasada artesanalmente como especie vegetal aislada y no en mezcla.

Adicionalmente, en el año 2016, se desarrolló una guía para la recolección de plantas medicinales nativas, a través de la mesa para la conservación y manejo de plantas medicinales tradicionales de la Araucanía ${ }^{(10)}$, cuyo propósito fue entregar recomendaciones generales para el proceso de recolección, solicitud de permisos y planificación de la recolección y alcances referidos a la selección, colecta, manejo y disposición de las partes de plantas cosechadas en el sur de Chile, incluyendo aspectos culturales de la cosmovisión y medicina tradicional mapuche. En ese sentido, se entrelazan las buenas prácticas de recolección, asociadas a la conservación de la biodiversidad, con la cultura y tradición ancestral de la Región de la Araucanía, lo que podría ser un primer acercamiento a una mirada ética biocultural en el uso terapéutico de las plantas medicinales.

Con relación a los aspectos éticos, en la formación de enfermería, el futuro profesional va adquiriendo conocimientos sustentados por fundamentos teóricos técnico-científicos, humanísticos y éticos, que le permiten desempeñarse con calidad y conciencia profesional. En esta misma línea, Escobar y $\mathrm{Cid}^{(11)}$ señalan que la enfermera debe demostrar una cultura ética y profesional para promover situaciones de bienestar, accesibilidad y eficacia en el cuidado de enfermería, y salvaguardar, en todo momento, los principios que la rigen, como son la solidaridad, integridad, equidad y calidad. No obstante lo anterior, la literatura establece que el desconocimiento por parte de los profesionales sanitarios podría generar dificultades en la prescripción y en la entrega de información a los pacientes sobre las plantas medicinales ${ }^{(12)}$.

Acorde a lo expuesto, el presente estudio tiene como propósito describir las representaciones sociales de las enfermeras comunitarias sobre los aspectos éticos y legales en el uso terapéutico de las plantas medicinales, como parte de los cuidados de enfermería en el contexto de atención primaria.

\section{MATERIAL Y MÉTODOS}

Estudio cualitativo, descriptivo, delineado por medio de las Representaciones Sociales (RS) en su perspectiva procesual, cuyo énfasis se coloca en el proceso social. Acorde a Banch ${ }^{(13)}$, para acceder al conocimiento de las RS con enfoque procesual se debe partir de un abordaje hermenéutico, entendiendo al ser humano como productor de sentidos, y focalizándose en el análisis de las producciones simbólicas, de los significados, del lenguaje, a través de los cuales los seres humanos construimos el mundo en que vivimos.

El colectivo estuvo constituido por las enfermeras/os de los centros de salud familiar de la comuna de La Serena, Chile. El diseño muestral fue intencional, y los criterios de inclusión fueron enfermeras con más de dos años de experiencia en APS. Los directores de los CESFAM, con consentimiento de las enfermeras, proporcionaron los contactos y la investigadora les consultó, de manera personal y por correo electrónico, su consentimiento de participación en el estudio, asimismo si deseaban participar en una entrevista individual o grupal. Se incorporaron a la muestra las enfermeras que decidieron participar y cuya representatividad se determinó por saturación de categorías $(n=22)$, es decir, por repetición de ideas, o evidencias suficientes para garantizar la credibilidad de la investigación.

Los métodos de producción de información fueron la entrevista focalizada individual (ocho participantes) y dos focus group (14 participantes), con una pauta para asegurar que todos los temas fueran explorados. Como apoyo para recoger la información se empleó una grabadora de audio, y se utilizó el método de observación y se registraron las 
impresiones mediante una bitácora. Las entrevistas individuales se efectuaron en un lugar externo al CESFAM, escogido por la misma entrevistada. Los focus group se realizaron en las dependencias del CESFAM, en un lugar acondicionado para este fin en un horario liberado para las enfermeras. Los datos se obtuvieron entre los meses de septiembre del año 2017 y agosto del año 2018. La validación de la información se efectuó mediante la triangulación de fuente, en la cual los investigadores, para confirmar los hallazgos y revisar algunos datos particulares, volvieron a los participantes durante el análisis de la información. También se utilizó la triangulación de datos, que consistió en efectuar comparaciones entre los datos obtenidos en las entrevistas individuales con los datos del grupo focal y la observación. Para el análisis de la información se efectuó el análisis de contenido cualitativo inductivo ${ }^{(14)}$, que consiste en profundizar más allá del contenido manifiesto de las entrevistas, considerando el contexto y el contenido latente donde se expresa el mensaje. Fue inductivo, ya que se caracterizó por una construcción de categorías emergentes desde el contenido, y que por tanto, se podían inferir.

Las/os participantes firmaron el consentimiento informado y el estudio fue aprobado por el Comité Ético Científico (Código: PI1713) de la Universidad de La Serena, Chile.

\section{RESULTADOS}

Del total de entrevistadas/os dieciocho fueron mujeres y sólo cuatro hombres; las edades fluctuaron entre veintiséis hasta sesenta y dos años. Los años de experiencia laboral en atención primaria variaron desde los tres hasta veintisiete años laborales. Entre las líneas temáticas, implicancias legales y aspectos éticos, se encontraron seis categorías que se presentan a continuación:

\section{Implicancias legales en el cuidado con plantas medicinales. En esta dimensión se desarrollaron} 3 categorías: Políticas públicas, Prescripción de plantas medicinales y Calidad de los fitofármacos.

Respecto a la categoría Políticas públicas, las entrevistadas se refirieron al escaso desarrollo de estas para respaldar el quehacer de enfermería y que estas permitan establecer lineamientos en la utilización de plantas medicinales. En el área de salud, es común que el quehacer de los equipos sanitarios se establezca en base a las políticas públicas, que se manifiestan de manera práctica a través de normas, protocolos, guías clínicas, metas sanitarias, entre otros. A pesar de la existencia de un manual de los medicamentos herbarios tradicionales, desde la perspectiva de las entrevistadas, es poco conocido a nivel de la atención primaria:

"Como funcionarios de salud del sistema público, por lo general, tenemos siempre que guiarnos por una indicación o una orden desde más arriba, ya sea ministerial o a nivel nacional. Algunas colegas quizás tengan ganas de cuidar con hierbas medicinales, y que también tengan los conocimientos, pero si no hay una orientación técnica, o una norma que te avale, que te respalde, porque no sé, supongamos un ejemplo, yo le indico ruda al paciente, y el paciente se intoxicó con eso, ¿quién te va a respaldar al respecto de este tema?, una colega puede tener todo el conocimiento, pero si no hay una norma legal o algo que te esté respaldando, yo creo que nadie se va a arriesgar hacerlo, yo siento que desde el ministerio de salud debe salir una norma, un protocolo, una orientación, algo" (Grupo focal 2).

"Necesitamos leyes que nos respalden, que den cuenta de nuestro quehacer y este tema de las terapias complementarias aún no está definido desde el código sanitario, está al debe, falta mucho por avanzar, faltan normas técnicas que nos señalen, por ejemplo, las dosis de las hierbas que podamos indicar, las veces que las debe tomar el paciente" (Entrevistada 1).

Otro tema relevante que emergió fue la Prescripción de plantas medicinales asociado a los aspectos legales en el cuidado con plantas medicinales es la prescripción enfermera. En el caso de Chile, no existe una ley que permita al profesional de enfermería generar una prescripción de medicamentos, menos aún de otros compuestos que no están definidos como medicamentos en la legislación nacional. Lo anterior cuestiona el quehacer de enfermería respecto a la indicación de plantas medicinales para efectuar cuidados:

"Hay una controversia ahi, porque si pensamos en la hierba igual tiene un principio activo, y si le indicaste una pomada de hierbas, ya es un fármaco que le estas indicando al paciente, pero sólo se lo dices verbalmente, entonces la indicación no queda en ninguna parte, sólo entre uno y el paciente" (Grupo focal 2).

"Generalmente para episodios agudos que tienen 
los pacientes, les indico alguna hierbita como para el dolor de estómago, cosas así, patologías simples, pero no queda anotado en la ficha por ejemplo, a lo más se lo anoto en un papel para que el paciente no se olvide del nombre de la hierba, como matico, el orégano, cosas asi, pero no queda registrado en un documento formal, como una receta o algo asi”" (Entrevistada 5).

En la categoría "Calidad de fitofármacos", estos son percibidos por las entrevistadas como productos que no demuestran calidad y efectividad como los medicamentos alópatas, dado principalmente a que no existe una legislación que fiscalice la preparación y envasado de estos productos. En Chile se definen fitofármacos como aquellas especialidades farmacéuticas cuyos ingredientes activos provienen de las partes aéreas o subterráneas de plantas $\mathrm{u}$ otro material vegetal y están debidamente estandarizados ${ }^{(15)}$. Los discursos de las entrevistadas manifiestan una desconfianza sobre el origen de los productos fitofármacos, señalando que muchos de estos productos se venden de manera informal:

"Cuando voy a comprar productos naturales en base a hierbas medicinales, pienso que me están estafando, quien me asegura a mi que realmente tiene el componente que dice el frasco. Cuando voy a comprar al mercado, me las venden muy baratas, como a tres mil o cuatro mil pesos, entonces yo me cuestiono, por ejemplo ¿será espirulina?, me entiendes, porque no es fácil producir la espirulina en gran cantidad, entonces me producen desconfianza, todo lo que yo veo en estos productos es que no vienen certificados, yo no los consumo, soy muy desconfiada en estos productos" (Entrevistada 3).

"En todas partes tú ves estos productos naturales, y las personas compran bastante, sobre todo los productos que son para adelgazar, pero esos productos ni siquiera están fiscalizados, entonces pueden estar ocasionando hasta efectos adversos o secundarios en los pacientes, es bastante complejo, a veces me dicen los pacientes: "señorita estoy tomado estas pastillas para bajar de peso, pero son naturales", pero categóricamente les señalo que no las deben consumir si no hay una indicación médica de respaldo, además las personas piensan que porque son naturales no les va a pasar nada malo al consumirlas" (Entrevistada 4).

2. Aspectos éticos en el cuidado con plantas medicinales. En esta dimensión emergieron tres categorías: Cuidado complementario, Cuidado con evidencia y Educación a la comunidad.

Respecto a la categoría Cuidado complementario, todas las entrevistadas coinciden que cuidar al paciente con plantas medicinales debe ser complementario al tratamiento farmacológico. En los relatos se concuerda en que la condición de salud del paciente debe ser determinante al momento de usar las plantas medicinales, es decir, que la persona se encuentre estable con su situación de salud o su enfermedad que la aqueja:

"Bajo ciertas condiciones del usuario, es decir, que estuviera bien controlado, que se tome sus medicamentos alópatas, y que viniese a las atenciones con la enfermera o con el médico, entonces ahí recién le podriamos ir agregando algún uso de hierba para complementar su tratamiento" (Grupo focal 2).

"El paciente debe estar completamente compensado para poder recién indicar algún complemento en base a hierbas medicinales, si el paciente es hipertenso o diabético, y no se toma sus medicamentos, y si además tiene los niveles altos de glicemia, podría empeorar su situación, a veces hay algunos pacientes que empiezan a tomar por ejemplo, la pata de vaca, pero están muy descompensados y no se dan cuenta, podría ser muy peligroso para su salud" (Grupo focal 1).

Acorde a lo expuesto, se establece que las entrevistadas consideran que no es apropiado que los pacientes con enfermedades crónicas dejen su tratamiento médico habitual, y que prioricen lo establecido por sus conocimientos adquiridos.

En la categoría "Cuidado con evidencia", emerge la necesidad de investigación y de evidencia científica sobre los beneficios de las plantas medicinales para la salud de las personas. La falta de evidencia es considerada una piedra angular al momento de articular estos productos con los cuidados. También se manifiesta el valor que se otorga desde el ámbito sanitario a la evidencia desde la investigación clínica. Ninguno/a de los/ las participantes manifestó interés sobre otros tipos de investigación, sino más bien se enfocaban en investigaciones de tipo experimental:

"Si una hierba tiene un estudio, una investigación, que señale que tiene beneficios para la salud, obviamente va a ser bueno ocuparlo, pero siempre con un conocimiento, simplemente eso, porque si yo indico algo, y no sé qué efectos tiene o qué beneficios tiene, entonces no sería ético indicarlo" (Entrevistada 6).

"Pienso que indicar aguas de montes requiere 
de un estudio más profundo, a nivel experimental, y que yo pueda ver efectivamente que hacen bien al paciente. Para indicar tiene que haber conocimiento te fijas, pero eso todavía no ha ocurrido con los montes" (Entrevistada 3).

Una categoría relevante, que consideraron las entrevistadas, fue la Educación a la comunidad sobre plantas medicinales. Todos los relatos de las entrevistadas en el ámbito educacional coinciden en un enfoque preventivo más que promocional, destacando la necesidad de educar en evitar efectos adversos y negativos de las plantas medicinales:

"Absolutamente creo que la educación es justa y necesaria, sobre todo en este momento cuando hay una invasión de información por redes sociales sobre estos temas, toda la gente comparte información sobre las plantitas que son buenas para la salud, pero no hay una educación sobre el uso seguro, entonces nosotras como profesionales de la salud sabemos que aunque sea sólo una plantita tienen sus efectos, y que pueden ser tan marcadas como los medicamentos, entonces como enfermeras tenemos el deber de estar educando continuamente a la gente" (Entrevistada 1).

"Las enfermeras siempre debemos educar a la población, es nuestro rol principal, pero creo que falta que salgamos más a la comunidad, estamos muy encerradas en el box. Es nuestro deber educar a la población sobre este tema de las hierbas medicinales $y$ productos naturales, debemos señalar sus efectos adversos, explicar por qué no debe ser automedicado, y señalar que es importante que le indiquen a su médico o enfermera lo que están consumiendo" (Grupo focal 1).

\section{DISCUSIÓN}

Las entrevistas permitieron arrojar ciertas categorías que finalmente permiten dar una aproximación acerca de las representaciones sociales sobre el tema planteado. Los resultados no son generalizables y solamente representan al grupo participante en el contexto de los cuidados de enfermería en APS. Es importante acotar que los resultados dan cuenta del parecer y sentir frente a estos desde un punto de vista subjetivo, es decir, desde sus propias construcciones, valores y criterios para evaluar los acontecimientos sobre el cuidado con plantas medicinales.
En la dimensión Implicancias legales en el cuidado con plantas medicinales y sus tres categorías, uno de los elementos centrales que señalan las entrevistadas, es el escaso desarrollo de Políticas públicas que respalden el quehacer de enfermería, y que estas permitan establecer lineamientos en la utilización de plantas medicinales. En el área de salud es común que el quehacer de los equipos sanitarios se establezca en base a las políticas públicas, que se manifiestan de manera práctica a través de normas, protocolos, guías clínicas, metas sanitarias, entre otros. A pesar de que existen algunas políticas y orientaciones respecto al uso de plantas medicinales y terapias complementarias en chile, como por ejemplo la norma técnica $\mathrm{N}^{\circ} 133 / 2012$ sobre el listado de medicamentos herbolarios tradicionales $^{(7)}$, y del decreto $\mathrm{N}^{\circ} 5 / 2013^{(16)}$ que reconoce el naturismo como profesión auxiliar de la salud, estas aún siguen siendo insuficientes para respaldar la praxis de enfermería. En el caso de Brasil, la medicina tradicional y complementaria está contemplada por el Sistema Único de Salud (SUS) desde la década de 1980, y en el año 2006 se estableció la Política Nacional sobre Prácticas Integrativas y Complementaria, que legitima la oferta pública de diferentes terapias, y que también permite a los profesionales no médicos practicar la medicina complementaria a través de la acreditación y remuneración por parte del SUS ${ }^{(17)}$. En este sentido, se podría establecer que el desarrollo de políticas públicas en esta temática podría favorecer la aplicación de las prácticas terapéuticas en el cuidado de enfermería, en busca de la plenitud de la asistencia, el bienestar de la población y el fortalecimiento de la atención primaria.

Respecto a la prescripción de plantas medicinales, en Chile, el código sanitario no establece esta actividad como parte del quehacer de enfermería. Este aspecto se convierte en una piedra angular al momento de incorporar las plantas medicinales en el cuidado, dado que las entrevistadas se cuestionan la prescripción al momento de indicar estos productos, expresando la necesidad de tener un respaldo legal para el cuidado. Lo anterior coincide con lo establecido por Contreras y Martínez ${ }^{(18)}$, quienes señalan que la prescripción enfermera está basada en los principios de subsidiariedad y sostenibilidad, mejorando el cuidado de la persona sin poner en riesgo su seguridad, favoreciendo el acceso a los pacientes a terapias farmacológicas y 
otros productos, y además hace mejor uso de las habilidades y competencias profesionales de la enfermera.

En la categoría Calidad de los fitofármacos, los discursos de las entrevistadas manifiestan una desconfianza sobre el origen de los productos fitofarmacológicos, señalando que muchos de estos se venden informalmente, y que no debiesen ser indicados por la enfermera. Es muy probable que, acorde a los relatos de las entrevistadas, se estén enfocando a los "fitofármacos artesanales", cuya producción, muchas veces, no es fiscalizada por un ente sanitario. Esta percepción coincide con la necesidad de establecer, por un lado, una mayor fiscalización de estos productos y, por otro, desarrollar y fortalecer acciones de fitovigilancia en los sistemas de salud ${ }^{(19)}$.

En la dimensión Aspectos éticos para el cuidado con plantas medicinales, en la categoría Cuidado complementario, las entrevistadas manifestaron que las plantas medicinales deben ser consideradas como complemento a la terapia convencional, y que no debe constituir un remplazo del tratamiento farmacológico de las enfermedades crónicas, dado principalmente por las interacciones que pudiesen ocurrir y por las complicaciones de la enfermedad cardiovascular que se pueden suscitar por la suspensión del tratamiento. Ninguna de las entrevistadas visualizó en sus relatos la posibilidad de reemplazar el tratamiento farmacológico alópata por una terapia tradicional o complementaria. Contrariamente a lo referido por las entrevistadas, se ha señalado que las personas acuden a las terapias tradicionales o complementarias, dado a que existe una mayor preocupación por parte de la ciudadanía sobre los efectos colaterales o adversos de los fármacos existentes ${ }^{(20)}$. Esta situación se correlaciona con los deficientes resultados que algunos pacientes han tenido a través de los tratamientos alópatas, lo que conlleva, por parte de los afectados, a buscar nuevas alternativas para tratar y sobrellevar una enfermedad o un malestar. $\mathrm{Al}$ respecto, un estudio realizado en $\mathrm{Brasil}^{(21)}$, encontró que el uso de plantas, como práctica de cuidado personal, tenía sus propios significados, tales como ayudar a los demás, cuidar a la familia, comodidad y una sensación de bienestar. En esta misma línea se estableció que los profesionales de la salud pueden mejorar la atención al insertarse en las realidades locales con el objetivo de establecer una alianza terapéutica con sujetos sociales y colectivos que reduzca las distancias entre el conocimiento académico y el popular. Se señala la necesidad de que el profesional apoye las prácticas de las personas en el cuidado con plantas medicinales, a través de coparticipación, respeto y apreciación del conjunto de conocimientos y experiencias, considerando los deseos y necesidades de la persona atendida como principios éticos de cuidado.

Respecto a la categoría Cuidado con evidencia, para las entrevistadas, una de las principales dificultades para cuidar con plantas medicinales es la falta de evidencia científica de estos productos, especialmente de las plantas típicas del sector. Esto coincide con una investigación realizada en Brasil $^{(17)}$, que señala un bajo número de estudios sobre medicamentos o tratamientos tradicionales y complementarios, estableciendo la necesidad de una pluralidad científica que valide los modos de conocimiento basados en la investigación cualitativa, incluida la percepción del individuo, para subsidiar la comprensión sobre la relación enfermera-paciente y la repercusión de esto en los resultados deseados. En este sentido, la investigación con enfoques cuantitativos pueden proporcionar una justificación teórica de estas terapias y garantizar la seguridad de las prácticas para los individuos.

En relación a la Educación a la comunidad, se establece como un área ética por parte de las entrevistadas, señalando que es de responsabilidad de la enfermería educar a la comunidad sobre promoción y prevención. Los discursos de las entrevistadas apuntan principalmente a una mirada preventiva en el uso de las plantas medicinales y productos naturales, dado a que la mayoría de las enfermeras refiere que es relevante educar sobre las contraindicaciones, efectos adversos e interacciones de las plantas con los medicamentos alópatas, coincidente con estudios realizados en Paraguay $^{(22)}$ y Costa Rica ${ }^{(23)}$, que establecen la necesidad de promover la cultura del uso seguro de la planta medicinal, y la entrega de información permanente acerca de la medicina tradicional, tanto a los consumidores como a los profesionales de la salud. Este aspecto sin duda es importante en tanto se quiera advertir a la población sobre los riesgos de las plantas medicinales. Ninguna de las entrevistadas mencionó la educación como una actividad promotora, o del uso de plantas 
medicinales como estrategia para fortalecer la medicina ancestral o tradicional de la comunidad, o como actividad para favorecer la autonomía en el cuidado de las comunidades, aun cuando algunas experiencias sobre la promoción de plantas medicinales realizadas en Brasil y Cuba dan cuenta de los beneficios sociales sobre este aspecto. En Brasil $^{(24)}$ se desarrolló un estudio cualitativo, cuyo propósito fue identificar los significados sobre el desarrollo de un jardín medicinal comunitario y se concluyó que elevar los conocimientos sobre plantas medicinales y cultura ambiental, en aquellos sectores que carecen de vías formales para adquirirlos, constituye un camino para construir comunidades sostenibles que convivan armónicamente con su entorno. En $\mathrm{Cuba}^{(25)}$ se realizó una investigación acción participativa, que incluyó una propuesta educativa a partir del cultivo de especies medicinales en recipientes para usar en espacios pequeńos en casa, como balcones, azoteas, terrazas, $\mathrm{u}$ otros sitios que se desee embellecer con un poco de jardín. Entre los principales resultados se demostró que esta actividad podía facilitar el cambio de estilos de vida, porque algunas especies medicinales son plantas multipropósitos que aportan tener a disposición, no solo medicamentos, sino también condimentos, porque muchas gustan frescas o secas para saborizar los alimentos y que además resultan apropiadas para sembrarlas con propósito ornamental. Los autores concluyeron que este tipo de actividades pueden proporcionar un gran impacto social y económico, aportando a la salud de la familia, que disfruta de una farmacia en el hogar y consume lo que allí se produce, con el consiguiente ahorro en la economía familiar.

Acorde a lo anterior, se establece la necesidad de orientar la actividad de promoción de la salud hacia estrategias que permitan el desarrollo y transformación de las comunidades, así como el desarrollo de huertas sustentables que incorporen las plantas medicinales como elemento relacional, de cultivo de saberes y de acceso a tratamientos naturales.

Se manifiesta el sentimiento y la necesidad de investigar en estas temáticas, pero los discursos, en este aspecto, no se separan del modelo occidental de generar ciencia, dado que se enfocan a la práctica basada en evidencia e investigación experimental. Ninguna de las entrevistadas manifiesta otras formas de investigación que abarquen aspectos culturales tal como la etnobotánica, etnografías, entre otros. En este aspecto, se evidencian las raíces de la formación de la profesión, donde se valora lo objetivo y lo comprobable, desde el punto de vista del método científico, dejando a un lado los saberes de las comunidades.

\section{CONCLUSIÓN}

En la dimensión Implicancias legales en el cuidado con plantas medicinales, se desarrollaron 3 categorías: Políticas públicas, Prescripción de plantas medicinales y Calidad de los fitofármacos y en la dimensión Aspectos éticos en el cuidado con plantas medicinales emergieron tres categorías: Cuidado complementario, Cuidado con evidencia y Educación a la comunidad.

Se observan tres aspectos centrales: El primero es el desarrollo de políticas públicas que apunten hacia el respaldo del accionar de la profesión, el segundo es el avance de la prescripción enfermera, que permita la autonomía de la profesión respecto al cuidado con plantas medicinales en la comunidad y el tercero es que los/las profesionales manifiestan que estas terapias asociadas a lo ancestral permiten cuidar desde una perspectiva holística, que es el núcleo del accionar y del pensar de la enfermería.

Los resultados de este estudio constituyen un primer avance en un ámbito poco explorado desde los cuidados de enfermería en la comunidad. En este sentido, futuras investigaciones podrían no sólo ampliar la muestra a otros contextos, sino también acceder a las prácticas que los profesionales llevan a cabo tanto en el ejercicio de la docencia como en el de la investigación.

\section{REFERENCIAS}

1. Cortés ME, Calderón F. Plantas medicinales chilenas: Desde el saber etnobotánico a los efectos terapéuticos y las reacciones adversas. Rev med Chile [Internet]. 2019 [citado 15 jul 2020]; 147(5): 673-674. Disponible en: https://scielo.conicyt. cl/scielo.php?script=sci_arttext $\&$ pid $=$ S003498872019000500673\&lng=es

2. Cuenca-Villalobos L, Uriarte-Sandoval M, Rodríguez-Díaz J, Bitanga M. Uso de la medicina no convencional por pacientes diabéticos. AMC [Internet]. 2020 [citado 28 jul 2020]; 
24(1): e6632. Disponible en: http://scielo.sld. $\mathrm{cu} /$ scielo.php?script=sci_arttext\&pid=\$102502552020000100008\&lng=es

3. Organización Mundial de la Salud (OMS). Medicina tradicional: definiciones [Internet]. [citado 02 ene 2019]. Disponible en: http://www. who.int/topics/tradicional_medicine/definitions/es/

4. Organización Mundial de la salud (OMS). Estrategia OMS sobre medicina tradicional 20142023 [Internet]. 2013 [citado 30 abr 2019]. Disponible en: https://apps.who.int/iris/bitstream/ handle/10665/95008/9789243506098_spa. pdf;jsessionid=F183527962D549EA9CE02EE8C 858CF 10 ? sequence $=1$

5. Gallegos-Zurita M. Las plantas medicinales: principal alternativa para el cuidado de la salud en la población rural de Babahoyo, Ecuador. An Fac Med [Internet]. 2016 [citado 31 may 2019]; 77(4): 327-332. Disponible en: http://www.scielo. org.pe/scielo.php?script=sci_arttext\&pid=S102555832016000400002\&lng=es.

6. Zeni A, Parisotto A, Mattos G, Tiaraju H. Utilizaçáo de plantas medicinais como remédio caseiro na Atenção Primária em Blumenau, Santa Catarina, Brasil. Ciênc saúde coletiva [Internet]. 2017 [citado 15 jul 2020]; 22(8): 2703-2712. Disponible en: http://www.scielo. $\mathrm{br} /$ scielo.php?script=sci_arttext $\&$ pid $=$ S141381232017002802703\&lng=es

7. Ministerio de Salud de Chile (MINSAL). MHT Medicamentos Herbarios Tradicionales. 103 especies vegetales [Internet]. 2009 [citado 3 ene 2018]. Disponible en: http://www.ispch.cl/sites/ default/files/decreto_exento_25.pdf

8. Instituto de Salud Pública, Chile. Definición de medicamento [Internet]. 2012 [citado 21 dic 2018]. Disponible en: http://www.ispch.cl/ anamed/subdeptoregistro/definicion

9. Biblioteca del Congreso Nacional (BCN). Resolución exenta 548 Aprueba listado de medicamentos herbarios tradicionales [Internet]. 2009 [citado 03 ene 2018]. Disponible en: https://www.leychile.cl/ Navegar?idNorma $=1005970$

10. Mesa para la Conservación y Manejo de Plantas Medicinales. Guía para la recolección de plantas medicinales nativas [Internet]. 2016 [citado 03 abr 2019]. Disponible en: https://cl.fsc.org/preview. gua-para-la-conservacin-de-plantas-medicinalesnativas.a-186.pdf

11. Escobar-Castellanos B, Cid-Henríquez P. El cuidado de enfermería y la ética derivados del avance tecnológico en salud. Acta Bioeth [Internet]. 2018 [citado 05 abr 2019]; 24 (1): 39-46. Disponible en: https://scielo.conicyt.cl/scielo.php?script=sci_ abstract\&pid=S1726-569X2018000100039\&lng= es\&nrm=iso

12. Mattos G, Camargo A, Sousa C, Zeni A. Plantas medicinais e fitoterápicos na Atenção Primária em Saúde: percepção dos profissionais. Ciênc saúde coletiva [Internet]. 2018 [citado 19 oct 2019]; 23(11): 3735-3744. Disponible en: http://www.scielo.br/scielo.php?script $=$ sci_ arttext\&pid=S1413-81232018001103735\&lng=es

13. Banch M. Aproximaciones procesuales y estructurales al estudio de las Representaciones Sociales. Papers on social representations [Internet]. 2000 [citado 3 abr 2018]; 9 (3): 1-15. Disponible en: http://psr.iscteiul.pt/index.php/PSR/article/ view/269/234

14. Díaz C. Investigación cualitativa y análisis de contenido temático orientación intelectual de revista Universum. Rev gen inf doc [Internet]. 2018 [citado 3 ene 2019]; 18(1): 119-42. Disponible en: https://revistas.ucm.es/index.php/RGID/article/ view/60813

15. Leighton A, Monsalve D. Documentación en terreno de buenas prácticas en fitoterapia en la red asistencial. MINSAL [Internet]. 2015 [citado 3 may 2019]. Disponible en: https://www.minsal. cl/wp-content/uploads/2015/09/INFORMEFINAL-FITOTERAPIA-30-12-2015.pdf

16. Ministerio de Salud de Chile. Reconocimiento al naturismo y regula la naturopatía como profesión auxiliar de la salud [Internet]. 2013 [citado $10 \mathrm{mar}$ 2019]. Disponible en: https://www.leychile.cl/ Navegar?idNorma $=1051691$

17. Azevedo C, Moura C, Corrêa H, Mata L, Chaves É, Chianca T. Prácticas integradoras y complementarias en el ámbito de la enfermería: aspectos legales y panorama académico-asistencial. Esc Anna Nery [Internet]. 2019 [citado 15 jul 2020]; 23(2): e20180389. Disponible en: http://www.scielo.br/scielo.php?script $=$ sci_ arttext\&pid=S1414-81452019000200226

18. Contreras V, Martínez J. Prescripción de enfermería en Chile. horiz enferm [Internet]. 2012 [citado 25 may 2019]; 23 (3): 32-39. Disponible en: http://horizonteenfermeria.uc.cl/23-3-2012/324prescripcion-de-enfermeria-en-chile-

19. De Carvalho M, Rossi F, Amaral M, Da Silva Luis V. Development of instrument to report and assess causality of adverse events related to herbal medicines. Vitae [Internet]. 2017 [citado 15 jul 2020]; 24(1): 13-22. Disponible en: http://www.scielo.org.co/scielo.php?script=sci arttext\&pid=S0121-40042017000100013\&lng=en

20. Lima C, Santos A, Messias R, Costa F, Barbosa D, Silva C. Prácticas integradoras y complementarias: utilización por agentes comunitarios de salud en el autocuidado. Rev Bras Enferm [Internet]. 2018 [citado 
14 jul 2019]; 71(Suppl 6): 2682-2688. Disponible en: http://www.scielo.br/scielo.php?script=sci_ arttext\&pid=S0034-71672018001202682\&lng=es

21. Badke M, Barbieri R, Ribeiro M, Ceolin T, Martínez-Hernáez À, Alvim A. Significados da utilização de plantas medicinais nas práticas de autoatenção à saúde. Rev esc enferm USP [Internet]. 2019 [citado 15 jul 2020]; 53: e03526. Disponible en: http://www.scielo.br/scielo.php?script=sci_ arttext\&pid=S0080-62342019000100494\&lng=en

22. Acosta-Recalde P, Zully Vera G, Morinigo M, Maidana G, Samaniego L. Uso de plantas medicinales y fitoterápicos en pacientes con Diabetes Mellitus tipo 2. Mem Inst Investig Cienc Salud [Internet]. 2018 [citado 15 jul 2020]; 16(2): 6-11. Disponible en: http://scielo.iics.una. py/scielo.php?script $=$ sci_arttext\&pid $=S 1812$ 95282018000200006\&lng=en

23. Hernández-Rivera P. Ingesta de productos naturales o macrobióticos: una llamada de atención a la salud pública. Enfermería Actual de Costa Rica [Internet]. 2017 [citado 06 jun 2019]; (33): 61-72. Disponible en: https://www.scielo.sa.cr/scielo.php?script=sci arttext\&pid=S140945682017000200061\&lng=es \&tlng=es

24. Costa C, García M, Ribeiro S, Salandini M, Bógus C. Hortas comunitárias como atividade promotora de saúde: uma experiência em Unidades Básicas de Saúde. Ciênc saúde coletiva [Internet]. 2015 [citado 07 jun 2019]; 20(10): 3099-3110. Disponible en: http://www.scielo.br/scielo.php?script=sci_ arttext\&pid=S141381232015001003099\&lng=es \&tlng=pt

25. Acosta L. Cultivo de plantas medicinales en recipientes. Rev Cubana Plant Med [Internet]. 2014 [citado 24 oct 2019]; 19(1): 4-13. Disponible en: http://scielo.sld.cu/scielo.php?script=sci_ arttext\&pid=S1028-47962014000100002\&lng=es 